\title{
Orthogonal polar spaces and unitals
}

\author{
Markus Stroppel
}

full screen

\section{Abstract}

We use the fact that certain classical unitals contain affine parts of orthogonal polar spaces in order to determine their full groups of automorphisms.

Keywords: hermitian form, quadratic form, polar space, unital, quaternion field, affine generalized quadrangle, affine polar space, automorphism.

MSC 2000: 51A50, 51E12, 51E24.

\section{Introduction}

We investigate a connection between suitable unitals and quadrics. This connection helps to determine the full group of automorphisms for hermitian unitals over commutative fields, or over quaternion fields if the form is trace-valued and the involution is the standard one, see Theorem 8.1 below. Thus our present approach extends the results in [14], where the characteristic two case and some of the non-commutative cases have been left open.

One of our tools will be the reconstruction of a hermitian form on a vector space $V$ from its restriction to the diagonal $\{(v, v) \mid v \in V\}$, cf. Section 4; here we extend an old result by Jacobson [8], see Remark 4.4.

\section{Hermitian forms and unitals}

Let $K$ be a (not necessarily commutative) field, and let $\sigma: K \rightarrow K: x \rightarrow \bar{x}$ be an involution: that is, an additive map with the properties $\overline{x y}=\bar{y} \bar{x}$ and $\overline{\bar{x}}=x$. In general, the set of fixed points of $\sigma$ is not closed under multiplication. However, we will consider a special case: 


Proposition 4.2 and Lemma 4.3 of the present section will be used below in Lemma 5.9 in order to reconstruct the hermitian form $k$.

Lemma 4.1. Let $K$ be either a quaternion field or a separable quadratic extension over $F$. For each $c \in K \backslash F$ with $T(c)=1$ we have $c-\bar{c} \neq 0$. Putting $j_{c}:=(c-\bar{c})^{-1}$ we obtain that $\varphi: x \mapsto T(x)-j_{c} c T(x)+j_{c} T(c x)$ describes the orthogonal projection from $K$ onto $F \oplus F c$. If $K$ is commutative this means $\varphi=\mathrm{id}$. If $K$ is not commutative we choose $p \in\{1, c\}^{\perp} \backslash\{0\}$ and obtain $x^{\varphi}+p^{-1}(p x)^{\varphi}=x$ for each $x \in K$.

full screen

close

Proof. The polar form $\beta_{N}$ is not degenerate on $C:=F+F c$, and $K$ is the orthogonal direct sum of $C$ and $C^{\perp}$. For any $p \in C^{\perp} \backslash\{0\}$ we have $C^{\perp}=p C=$ $C p$. The relations $\bar{c}=1-c$ and $p \perp C$ imply $p u=\bar{u} p$ for each $u \in C$. Now straightforward computations yield the formulae, as claimed.

Proposition 4.2. Let $V$ be a vector space over some quaternion field $H$, and let $h: V \times V \rightarrow H$ be a non-degenerate $\sigma$-hermitian form. Consider the quadratic $\operatorname{map}^{1} v \mapsto h(v, v)$ and the corresponding polar form

$$
f(v, w)=h(v, w)+h(w, v)=h(v, w)+\overline{h(v, w)}=T(h(v, w)) .
$$

As in Lemma 4.1 we choose $c \in H$ with $T(c)=1$ and $p \in\{1, c\}^{\perp} \backslash\{0\}$, and put $j_{c}:=(c-\bar{c})^{-1}$.

(a) For all $v, w \in V$ we have

$$
\begin{array}{rl}
h(v, w)=f(v, w)-j_{c} c & f(v, w)+j_{c} f(c v, w) \\
+p^{-1}\left(f(p v, w)-j_{c} c f(p v, w)+j_{c} f(c p v, w)\right) .
\end{array}
$$

(b) The form $f$ is non-degenerate.

(c) Every $H$-semilinear semi-similitude $\alpha \in \Gamma \mathrm{O}(f)$ is a semi-similitude of $h$.

Explicitly, assume that there exist some $\gamma \in \operatorname{Aut}(F)$ and $s \in F^{\times}$such that $f\left(v^{\alpha}, w^{\alpha}\right)=f(v, w)^{\gamma} s$ and let $\mu$ be the companion automorphism of $\alpha$. Then $\mu$ induces $\gamma$ on $F$ and $h\left(v^{\alpha}, w^{\alpha}\right)=h(v, w)^{\mu} s$.

Proof. The first assertion follows from Lemma 4.1 and the observation

$$
\begin{aligned}
h(v, w) & =h(v, w)^{\varphi}+p^{-1}(p h(v, w))^{\varphi} \\
& =f(v, w)-j_{c} c f(v, w)+j_{c} f(c v, w) \\
& \quad+p^{-1}\left(f(p v, w)-j_{c} c f(p v, w)+j_{c} f(c p v, w)\right) .
\end{aligned}
$$

\footnotetext{
${ }^{1}$ In general, the range of this quadratic map will consist of the set of fixed points of $\sigma$, and will not be contained in the center of $H$. However, the values of the polar form lie in $F$ by Assumptions 2.1 .
} 

Passing to inhomogeneous coordinates, we will identify the point $K(x, y, 1)$ with $(x, y) \in K^{2}$. This affine plane also carries the richer structure of an affine space

$$
\mathbb{A}:=\left(K^{2},\left\{a+F v \mid a \in K^{2}, v \in K^{2} \backslash\{0\}\right\}\right)
$$

over the smaller (and commutative) field $F$, which will be utilized in the sequel. The dimension of this affine space is $\operatorname{dim}_{F}\left(K^{2}\right)=2 \operatorname{dim}_{F} K \in\{4,8\}$, see Remark 2.4.

The projective hull $\mathbb{P}$ of $\mathbb{A}$ may be described by homogeneous coordinates $F(x, y, s)$, with $x, y \in K$ and $s \in F$. We will identify the hyperplane $\mathbb{A}_{\infty}:=\mathbb{P} \backslash \mathbb{A}$ at infinity with the projective space consisting of all $F$-subspaces of $S$.

We interpret the affine part $U \backslash b_{S}$ of the unital in terms of $\mathbb{A}$ :

Lemma 5.1. (a) The set $A:=U \backslash b_{S}=\{(x, y) \mid x \bar{y}+y \bar{x}+1=0\}$ is the affine part of the quadric $Q$ described by the quadratic form

$$
\begin{aligned}
q: K^{2} \times F \rightarrow F:(x, y, s) \mapsto k((x, y, s) & ,(x, y, s)) \\
& =x \bar{y}+\overline{x \bar{y}}+s^{2}=x \bar{y}+y \bar{x}+s^{2}
\end{aligned}
$$

with polar form $\beta_{q}((x, y, s),(u, v, t))=x \bar{v}+v \bar{x}+y \bar{u}+u \bar{y}+2 s t$.

(b) For all $v, w \in K^{2} \times F$, we obtain $q(v)=k(v, v)$ and

$$
\beta_{q}(v, w)=k(v, w)+k(w, v)=k(v, w)+\overline{k(v, w)} .
$$

The restriction $\left.q\right|_{S}$ is non-degenerate; its Witt index is $\operatorname{dim}_{F} K$.

Lemma 5.2. Every block $b$ of the unital that meets $b_{S}$ induces an affine subspace $X_{b}$ of $\mathbb{A}$ such that the completion of $X_{b}$ in $\mathbb{P}$ is totally singular with respect to $q$. That subspace either is a line (if $K$ is commutative) or has dimension 3 (if $K$ is a quaternion field).

Proof. Let $K(a, b, 1)$ and $K(x, y, 1)$ be affine points on a block of the unital; then $a \bar{b}+b \bar{a}+1=0=x \bar{y}+y \bar{x}+1$. The joining line $K(a, b, 1) \oplus K(x, y, 1)$ meets $S$ in the point $K(a-x, b-y, 0)$. This point belongs to the unital precisely if $0=(a-x) \overline{(b-y)}+(b-y) \overline{(a-x)}=a \bar{b}+b \bar{a}+x \bar{y}+y \bar{x}-(a \bar{y}+y \bar{a}+b \bar{x}+x \bar{b})$. This yields $a \bar{y}+y \bar{a}+b \bar{x}+x \bar{b}=a \bar{b}+b \bar{a}+x \bar{y}+y \bar{x}=-2$, and $\beta_{q}((a, x, 1),(b, y, 1))=$ $a \bar{y}+y \bar{a}+b \bar{x}+x \bar{b}+2=0$.

We have thus proved that any two affine points on the considered block are orthogonal with respect to $\beta_{q}$. Since points on the unital are singular, this means that the affine part of the block is contained in a totally singular subspace. Conversely, the affine part of this subspace is contained in the quadric (and thus in the unital), and coincides with the affine part of the block. 
In general, only a partial converse of Lemma 5.2 is true:

Lemma 5.3. Let $T$ be a line of $\mathbb{P}$ that is totally singular with respect to $q$. Then the intersection of $T$ with $\mathbb{A}$ is contained in a unique block of the unital that meets the block $b_{S}$.

Proof. Let $(a, b)$ and $(x, y)$ be two affine points that span $T$. Then we have $a \bar{b}+b \bar{a}+1=q(a, b, 1)=0=q(x, y, 1)=x \bar{y}+y \bar{x}+1$ and $0=q(a-x, b-y, 0)$ because the space $T$ is totally singular. The line $K(a, b, 1) \oplus K(x, y, 1)$ joining the two points in the projective plane over $K$ meets the line $S$ in the point $K(a-x, b-y, 0)$. This point belongs to the unital, and all affine points of $T$ lie in the block induced by $K(a, b, 1)+K(x, y, 0)$, as claimed.

Definition 5.4. We use the polar space $\mathcal{Q}$ (consisting of all totally singular subspaces of $K^{2} \times F$, with respect to $q$ ) in order to gain a deeper understanding of the unital. The affine part of $\mathcal{Q}$ will be denoted by $\mathcal{A}$.

If (and only if) $K$ is commutative, this affine part of the quadric forms an affine quadrangle with respect to a full weak subquadrangle: the maximal singular subspaces are lines. See [11] for an axiomatization of that class of geometries; cf. also [13] and [12]. We will denote the set of nonempty affine restrictions of maximal totally singular subspaces (with respect to $q$ ) by $\mathcal{M}$.

Remark 5.5. The lines in $\mathcal{Q}$ are maximal totally singular subspaces if, and only if, the field $K$ is commutative (i.e., if $\left.\operatorname{dim}_{F} K=2\right)$. In that case $\left(A, B^{S}\right)$ is equal to $(A, \mathcal{M})$. If $K$ is not commutative, pick two affine points $a, b \in Q$ that span a totally singular line $\ell$. Then the orthogonal space $\ell^{\perp}$ has $F$-dimension greater than 3, and contains affine points of $Q$ that do not belong to the block of the unital that joins $a$ and $b$. This shows that, in the non-commutative case, not every element of $\mathcal{M}$ is induced by a block of the unital.

We collect our results so far:

Theorem 5.6. (a) The quadratic form $q$ is non-degenerate; its Witt index equals $\operatorname{dim}_{F} K \in\{2,4\}$.

(b) The stabilizer Aut $(U, B)_{b_{S}}$ acts faithfully by automorphisms of $(A, \mathcal{M})$.

(c) If $K$ is commutative then the affine part $\mathcal{A}$ of $\mathcal{Q}$ consists of the affine subspaces (in $\mathbb{A}$ ) of the blocks of $U$ that meet the block $S$.

(d) If $K$ is not commutative then there are totally singular subspaces whose affine part is not contained in any block of the unital.

Proof. The quadratic form $q$ is non-degenerate because no point belongs to every totally singular subspace. From Lemma 5.2 and Lemma 5.3 we know that 



\section{Automorphisms of affine polar spaces}

The following is shown in [2, 2.7]:

Proposition 6.1. Every isomorphism between affine parts $\mathcal{A}_{j}=\mathcal{Q}_{j} \backslash \mathcal{H}_{j}$ of polar spaces $\mathcal{Q}_{1}$ and $\mathcal{Q}_{2}$ of rank at least 2 (obtained by deleting geometric hyperplanes $\mathcal{H}_{1}$ and $\mathcal{H}_{2}$, respectively) extends uniquely to an isomorphism between the polar spaces.

By Proposition 6.1 every automorphism of $\mathcal{A}$ extends to a unique element of $\operatorname{Aut}(\mathcal{Q})$. If $K$ is not commutative then the polar space $\mathcal{Q}$ can be recovered from the system $\mathcal{M}$ of all affine maximal totally singular subspaces:

Lemma 6.2. Every automorphism of $(A, \mathcal{M})$ extends uniquely to an automorphism of the polar space $\mathcal{Q}$ defined by $q$.

Proof. We claim that for each totally singular subspace $T$ of $\mathcal{Q}$ there is a set $M_{T}$ of maximal totally singular subspaces such that $A \cap T=\bigcap_{X \in M_{T}}(A \cap X)$.

In order to see this, choose first ${ }^{2}$ a subspace $V \leq K^{2} \times F$ such that $K^{2} \times F=$ $\left(K^{2} \times F\right)^{\perp} \oplus V$ and $T \leq V$; this is possible because the quadratic form is not degenerate. Now one may take $M_{T}$ as the set of all maximal totally singular subspaces contained in $T^{\perp} \cap V$. The radical of $T^{\perp} \cap V$ is just $T$, and $M_{T} / T:=\left\{X / T \mid X \in M_{T}\right\}$ is the set of all maximal totally singular subspaces of $Z:=\left(T^{\perp} \cap V\right) / T$, with respect to the induced quadratic form $\left.q\right|_{Z}$. As the polar form $\beta_{\left.q\right|_{Z}}$ is non-degenerate, we have $\{0\}=\operatorname{rad}\left(\beta_{\left.q\right|_{Z}}\right) \geq \bigcap_{X \in M_{T}} X / T$, and $T=\bigcap_{X \in M_{T}} X$ follows. Consequently, every automorphism of $(A, \mathcal{M})$ is an automorphism of the affine polar space $\mathcal{A}$. The extension to $\mathcal{Q}$ exists by Proposition 6.1.

Consider a non-degenerate quadratic or hermitian form of Witt index at least 2 on a vector space $V$ with $5 \leq \operatorname{dim} V<\infty$. Then every automorphism of the corresponding polar space is induced by a semi-similitude, cf. [6, 8.1.5]. Therefore, we have:

Corollary 6.3. Every automorphism of $(A, \mathcal{M})$ is induced by a semi-similitude of $q$ that leaves $\mathbb{A}_{\infty}$ invariant.

\footnotetext{
2 This precaution is necessary if char $K=2$ because then $\beta_{q}$ is degenerate; cf. [4, $\left.\S 16\right]$.
} 


\section{Extending automorphisms of the quadric}

The points at infinity for the affine plane over $K$ are obtained by identifying those points that belong to the same orbit under multiplication (from the left) with elements of $K^{\times}$. Let $\Sigma$ denote the group of automorphisms of the projective space $\mathbb{P}$ induced by maps of the form $(x, y, z) \mapsto(m x, m y, z)$, with $m \in K^{\times}$. Then the $\Sigma$-orbits are the classes $[X]_{\sim}$ with respect to the equivalence relation $\sim$ given by $F(a, b, 0) \sim F(x, y, 0) \Longleftrightarrow \exists m \in K^{\times}: F(a, b, 0)=F(m x, m y, 0)$.

full screen

close

quit

ACADEMIA PRESS

NWII

$\widehat{\overline{\text { IIIIIII }}}$

UNIVERSITEIT GENT

Theorem 7.1. An element $\gamma \in \operatorname{Aut}(\mathcal{A})$ induces an automorphism of the unital $(U, B)$ if, and only if, it normalizes $\Sigma$. In particular, not every automorphism of $\left(U \backslash b_{S}, B^{S}\right)$ extends to an automorphism of $(U, B)$.

Proof. Recall from Proposition 6.1 and Corollary 6.3 that $\gamma$ is induced by a semi-similitude of $q$, that is, by an element of $\Gamma \mathrm{O}(q)$. Assume first that $\gamma$ normalizes $\Sigma$. Then $\gamma$ respects the relation $\sim$ on $\mathbb{A}_{\infty}$. Thus the action of $\gamma$ extends to an action on the projective plane over $K$, induced by a $K$-semilinear map in $\Gamma \mathrm{O}(\tilde{q})$. According to Proposition 4.2, this semilinear map is a semisimilitude of the hermitian form $f$. Thus it leaves $U$ invariant, and respects the blocks because these (apart from $b_{S}$ ) are obtained by joining a point of $A$ with $[X]_{\sim}<\mathbb{A}_{\infty}$, for some $X \in Q \cap \mathbb{A}_{\infty}$.

Now assume that $\gamma$ induces an automorphism of the unital. Then $\gamma$ preserves the relation $\sim$. This means that the group $\gamma^{-1} \Sigma \gamma$ has the same orbits as $\Sigma$ on $K^{2} \times\{0\}$. We also know that $\gamma^{-1} \Sigma \gamma$ is induced by a subgroup of $\mathrm{O}\left(q^{\prime}\right) \times\{\mathrm{id}\}$ because the latter is a normal subgroup of $\operatorname{Aut}(\mathcal{A})$, cf. Corollary 6.3. Looking at the images of $F(1,0,0), F(0,1,0)$ and $F(1, y, 0)$ under an arbitrary element $\varphi \in \gamma^{-1} \Sigma \gamma$ we find that $\varphi$ is induced by a map of the form $(x, y, z) \mapsto\left(x^{\alpha}, y^{\beta}, z\right)$, where $\alpha$ and $\beta$ are $F$-linear bijections of $K$ such that

$$
\forall s \in K^{\times} \forall y \in K \exists t \in K^{\times}:\left(s^{\alpha},(s y)^{\beta}\right)=(t, t y) .
$$

Specializing $y=1$, we find $\alpha=\beta$. Considering the general case again, we obtain $s^{\alpha} t=(s t)^{\alpha}$ for all $s, t \in K$. This means that $\alpha$ centralizes the multiplications by elements of $K$ from the right. Thus $\alpha$ is contained in the group of multiplications by elements of $K^{\times}$from the left, see [10, 4.4, Lemma 2]. We have proved that $\gamma$ normalizes $\Sigma$.

Example 7.2. There is no chance to reconstruct the relation $\sim$ inside the affine part of the polar space: Let $\alpha$ be an $F$-linear bijection of $K$. Since $K \times\{0\} \times\{0\}$ and $\{0\} \times K \times\{0\}$ are totally singular subspaces of $S$, there exists a unique $F$-linear bijection $\widehat{\alpha}$ such that $(x, y, 0) \mapsto\left(x^{\alpha}, y^{\widehat{\alpha}}, 0\right)$ gives an element $\alpha^{\prime} \in \mathrm{O}\left(q^{\prime}\right)$. For a suitable choice of $\alpha$, we find that $\alpha^{\prime}$ does not preserve the equivalence relation $\sim$. We give explicit examples: 


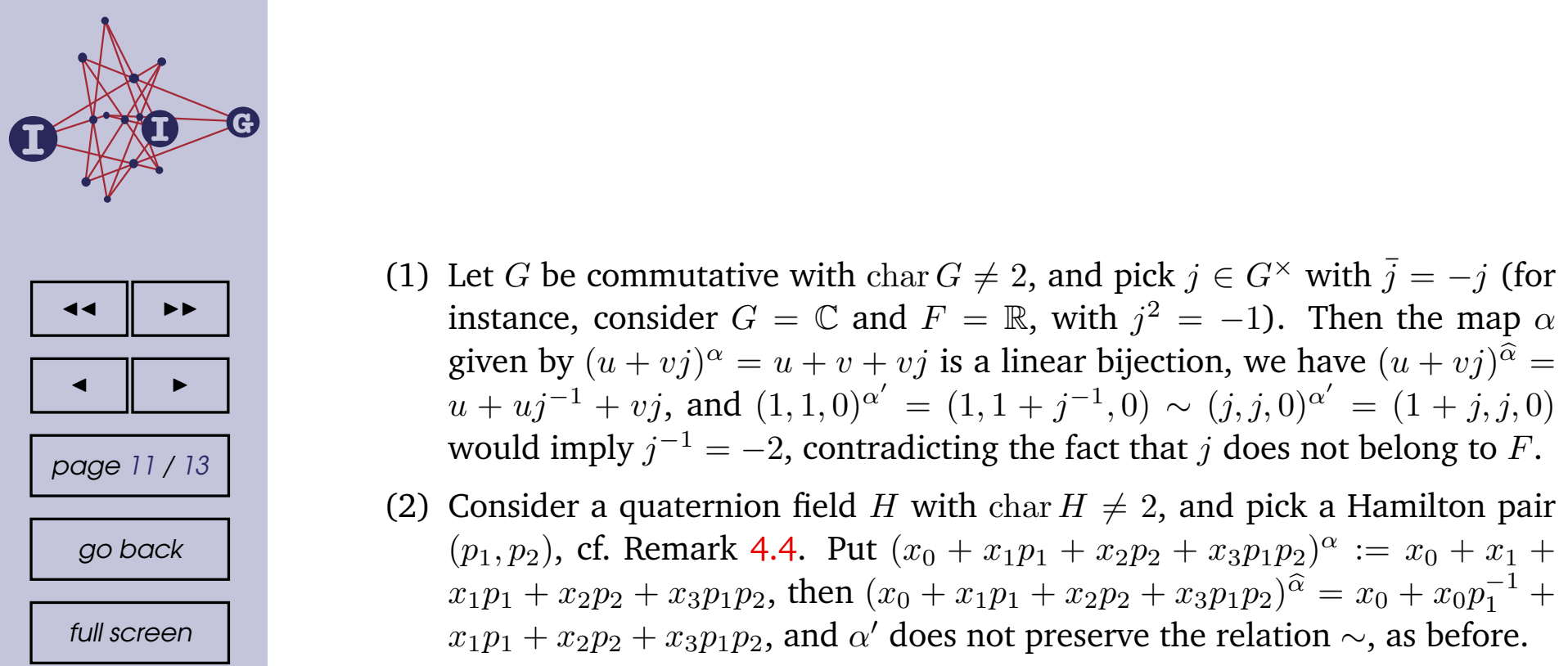

\section{Automorphisms of the unital}

From Theorem 5.6(b) we know that every automorphism $\varphi$ of $(U, B)$ that leaves $b_{S}$ invariant induces an automorphism of $(A, \mathcal{M})$. Since the action of $\varphi$ on $\mathcal{A}$ extends to a unique action on $(U, B)$ and to a unique action on the polar space $\mathcal{Q}$ (cf. Lemma 6.2), we have an injective group homomorphism $\varphi \mapsto \widehat{\varphi}$ from the stabilizer Aut $(U, B)_{b_{S}}$ into the stabilizer $\operatorname{Aut}(\mathcal{Q})_{Q_{\infty}}$.

From Corollary 6.3 we know that $\operatorname{Aut}(\mathcal{Q})_{Q_{\infty}}$ is induced by the stabilizer of $S$ in the group $\Gamma \mathrm{O}(q)$, which in turn induces the group $\Gamma \mathrm{O}\left(q^{\prime}\right)$ on $S$. According to Theorem 7.1, the image of Aut $(U, B)_{b_{S}}$ under the homomorphism - induces the normalizer of $\Sigma$ in $\Gamma \mathrm{O}\left(q^{\prime}\right)$. This normalizer consists of the $K$-semilinear semi-similitudes of $q^{\prime}$, and these extend to semi-similitudes of the hermitian form $k$ by Lemma 4.3, cf. Lemma 5.9.

Since the group of (semi-)similitudes of the hermitian form $k$ acts transitively on the set $B$ of blocks of the unital, the full group of automorphisms of the unital is obtained as the product of $\mathrm{PU}(k)$ and the stabilizer of $b_{S}$. As we have just seen, this stabilizer is induced by semi-similitudes, and we have proved the following.

Theorem 8.1. Every automorphism of the unital is induced by a semi-similitude of the hermitian form; we have Aut $(U, B)=\mathrm{P \Gamma U}(k)$.

A translation with center $p$ of a unital is an automorphism fixing every block through $p$. The group $\mathrm{T}$ generated by all translations is called the little projective group of the unital.

Corollary 8.2. The little projective group $\mathrm{T}$ is normal in $\mathrm{Aut}(U, B)$, and the latter acts faithfully by conjugation on T. Thus Aut $(U, B)$ is embedded in Aut $(\mathrm{T})$.

Remark 8.3. Once we know $\operatorname{Aut}(U, B)=\mathrm{P} \Gamma \mathrm{U}(k)$ it is easy to compute the translations with center $p$; they form a subgroup of the (abelian) group of elations with center $p$ and axis $p^{\perp}$ in the projective plane over $K$. Thus $\mathrm{T}$ is the 

2nd edition. , Basic algebra. II, W. H. Freeman and Company, New York, 1989,

[11] H. Pralle, Affine generalized quadrangles—an axiomatization, Geom. Dedicata 84 (2001), no. 1-3, 1-23, doi:10.1023/A:1010300704221.

[12] N. Rosehr, Topological affine quadrangles, Innov. Incidence Geom. 1 (2005), 143-169.

www . mathematik . uni-wuerzburg. de/ rosehr/preprints/TopAffQuad.pdf

[13] B. Stroppel, Point-affine quadrangles, Note Mat. 20 (2000/01), no. 1, $21-$ 31, doi:10.1285/i15900932v20n1p21.

[14] M. Stroppel and H. Van Maldeghem, Automorphisms of unitals, Bull. Belg. Math. Soc. Simon Stevin 12 (2005), no. 5, 895-908. http://projecteuclid.org/euclid.bbms/1136902624

Markus Stroppel

Fachbereich Mathematik, Universität StUttgart, D-70550 StUtTGaRT, GERMANY

e-mail: stroppel@mathematik.uni-stuttgart.de 